\title{
Reviewer Acknowledgements for Global Journal of Health Science, Vol. 9, No. 8
}

Global Journal of Health Science wishes to acknowledge the following individuals for their assistance with peer review of manuscripts for this issue. Their help and contributions in maintaining the quality of the journal are greatly appreciated.

Global Journal of Health Science is recruiting reviewers for the journal. If you are interested in becoming a reviewer, we welcome you to join us. Please find the application form and details at http://recruitment.ccsenet.org and e-mail the completed application form to gjhs@ccsenet.org.

\section{Reviewers for Volume 9, Number 8}

Amy E. Jetton, Middle Tennessee State University Murfreesboro, United States

Angus N Oli, Nnamdi Azikiwe University, Nigeria

Arpad Kovacs, University of Kaposvar, University of Pecs, Hungary

Ayesha Johnson, University of South Florida, United States

Basak Baglama, Near East University, Cyprus, Cyprus

Clauden Louis, University of Rochester Medical Center, United States

Delfina Gabriela Ramos, Polytechnic Institute of Cávado and Ave, Barcelos, Portugal

Dongxu Fu, University of Virginia, United States

Evangelia Mavrikaki, National \& Kapodistrian University of Athens, Greece

Evanthia Sakellari, Technological Educational Institute of Athens, Greece

Farahnaz Amini, UCSI University, Malaysia

Gavric Zivana, Professor, Ph D MD, Bosnia and Herzegovina

Georgann Valerie Weissman, Capella University, United States

Hilal Alrahbi, University of Nizwa, Oman

Hülya YARDIMCI, Ankara University, Turkey

Jaime Hinzpeter, Clinical Hospital University of Chile, Chile

Jose R. Cordon, Universidad de Cadiz (Spain), Spanish Goverment, Spain

Kartheek R Balapala, University Tunku Abdul Rahman, Malaysia

Loray Daws, British Columbia Masterson Institute, Canada

Marcel Wullschleger, University of Bern, Switzerland

Matteo Vitali, Hygiene at Sapienza University, Italy

Monia Ouederni, University Tunis El Manar Tunisia, Tunisia

Nant Thin Thin Hmwe, University Malaysia Sabah, Malaysia

Pi-Ming Yeh, Missouri Western State University, United States

Raymond Compton Jagessar, University of Guyana, Guyana

Regina E.Ella, University Of Calabar, Nigeria

Rhoda Suubi Muliira, University of Buraimi, Al Buraimi, Oman

Roger Ho, Yong Loo Lin School of Medicine, National University of Singapore, Singapore

Roman Levytskyy, University of Nebraska-Lincoln, United States

Ru-Jeng Teng, Medical College of Wisconsin, United States

Srikrishna Sulgodu Ramachandra, Public Health Foundation of India, India 
Thomas Wolfgang Leiblein, Zurich University of Applied Sciences, Switzerland

Trisha Dunning, Deakin University and Barwon Health, Australia

Yasam Kemal Akpak, Ankara Mevki Military Hospital Department of Obstetrics and Gynaecology, Turkey

Younsook Yeo, St. Cloud State University, USA 